Neuroepidemiology 2013;41:217-218

DOI: $\underline{10.1159 / 000355128}$

\section{Yes, It Is Time to Reconsider How We Rate Cognitive Impairments in HIV Disease}

\author{
James T. Becker ${ }^{\mathrm{a}-\mathrm{c}}$, Beth E. Snitz ${ }^{\mathrm{b}}$ \\ Departments of a Psychiatry, ${ }^{\mathrm{b}}$ Neurology and ${ }^{\mathrm{C} P \text { Pychology, }}$ \\ University of Pittsburgh, School of Medicine, Pittsburgh, Pa., USA
}

Meyer et al. [1] have provided us with an excellent review, data analysis and proposal regarding criteria for cognitive impairments in HIV disease. They identified the effect of different individual criteria for defining impairment and the effects of different 'falsepositive' rates on classification. This discussion is critical for our understanding of the incidence, prevalence and temporal trends in $\mathrm{HIV}$-associated neurocognitive disorders (HAND) and is more generally relevant to any neurobehavioral syndrome without a clear antemortem biomarker to confirm the presence of a neuropathological process.

The analysis of any neurobehavioral syndrome naturally must occur on at least two levels, namely clinical and pathological. Although some diagnostic criteria (e.g. those for Alzheimer's disease) make this distinction explicit, in more common parlance the distinction between HAND (or Alzheimer's dementia) and the neuropathology of HIV disease (or Alzheimer's disease) may be lost. This is particularly problematic in HIV disease, as the neuropathological hallmarks of HIV encephalitis (the pathological correlate of HIV dementia), the combination of white matter pallor, perivascular chronic inflammation and multinucleated giant cells, are now rare. The search for antemortem biomarkers has been generally unsuccessful [2], likely reflecting the multiple pathways through which central nervous system dysfunction can occur in HIV disease. Until such time as we are able to establish a 'ground truth' with regard to the underlying causes of HAND, we are left with a clinical decision. This is where the paper of Meyer et al. [1] becomes so important.

The presence and impact of 'false positives' in neurocognitive disorders, particularly in the mild spectrum, receive scant attention from researchers. The likelihood of these errors is compounded by two common practices to increase sensitivity to ever milder neurocognitive abnormalities, namely (1) extensive test batteries, resulting in uncorrected multiple comparisons, and (2) higher cutoff scores, increasing the overlap between critical portions of test score distributions in individuals with and without disease. The 'cost' of increased sensitivity is necessarily a reduction of specificity, a problem rarely explored with regard to neurocognitive classification. Meyer et al. [1] have given us a most comprehensive and systematic investigation of the cost to specificity as a function of the number of cognitive tests, domains, cutoff scores, sample size, intertest correlations and other factors.

They conclude that the safest way to classify individuals is to use an average standard score across all of the measures in a cognitive domain, to lower the threshold for mild impairment to a $\mathrm{Z}$ score of $\leq-1.5$ and to limit the number of domains evaluated. We offer an additional consideration, namely that the classification of asymptomatic neurocognitive impairment may well be clinically irrelevant. By definition, individuals without symptoms will not seek medical attention. The individuals with asymptomatic neurocognitive impairment might thus represent the lower end of the normal distribution of function.

One of the difficulties of this discussion, however, is the apparent premise that there should be no degree of cognitive dysfunction among individuals without HIV infection. That is, all other things being equal, the goal appears to be to restrict 'false positives' to as close to 0 as is possible without sacrificing 'true positives', a feat made more difficult by the absence of a neuropathological standard. Unfortunately, as noted by Meyer et al. [1], there is a range of factors that can affect individuals' test performance, and that may result in mildly (or less frequently, severely) impaired cognitive function. The attribution of the cause of cognitive impairment was considered important even early in the HIV epidemic $[3,4]$ and is now part of the diagnostic classification scheme [5]. These non-HIV-related causes can include histories of drug and alcohol use, traumatic brain injury, affective disorders and the like. Thus, in addition to the problem of nondifferential misclassification, we also have a problem with the attribution of risk.

This problem becomes even more confounded in HIV disease as infected individuals enter middle and older age. In addition to the effects of HIV infection on brain structure and function, now the normal consequences of aging - such as hypertension, diabetes and vascular disease - begin to take their toll. Even assuming a relatively low rate of true HIV-related neuropathology for individuals over the age of 65 , for example $5 \%$, when added to the preexisting 'false-positive' rate (due to other pathologies, both ageand non-age-associated) this decreases even more the sensitivity to any HIV-related dysfunction and may minimize the detectable efficacy of any intervention. When these factors are coupled with the change in the treated history of individuals with HIV disease, so-called cohort effects, the attribution of risk and interpretation of interventions becomes even more complicated.

Given the complexity of the problem, some might argue that it is simply too much to deal with, and that the only solution is to either abandon clinical trials related to HAND or to focus entirely on preclinical models. We strongly reject this sort of argument because we reject the premise that the problems are insurmountable. Meyer et al. [1] have carefully explored one aspect of the problem, the classification of cognitive impairment, and they provide us

\section{KARGER}

(C) 2013 S. Karger AG, Base

0251-5350/13/0414-0217\$38.00/0
James T. Becker, PhD

Neuropsychology Research Program

Suite 830, 3501 Forbes Avenue

Pittsburgh, PA 15213 (USA)

E-Mail BeckerJT@upmc.edu 
with a specific target as they propose clear cutoff scores. This should lead to further investigation of the problem among larger cohorts that include neuropsychological evaluations, all of which should lead to greater clarity in interpreting the results of projects being completed in the post-highly active antiretroviral therapy era. This is but one step in one part of the process, but it is an important step. We are all very grateful to our colleagues for pushing us forward.

\section{Acknowledgements}

Preparation of the manuscript was supported in part by funds from the National Institutes of Health (AG034852, AI035041, MH098745, AG038479, AG05133).

\section{References}

1 Meyer ACL, Boscardin WJ, Kwasa JK, Price RW: Is it time to rethink how neuropsychological tests are used to diagnose mild forms of HIV-associated neurocognitive disorders? Impact of false-positive rates on prevalence and power. Neuroepidemiology 2013;41:208-216.

2 Price RW, Epstein LG, Becker JT, Cinque P, Gisslen M, Pulliam L, et al: Biomarkers of HIV-1 CNS infection and injury. Neurology 2007;69: 1781-1788.

-3 Nomenclature and research case definitions for neurologic manifestations of human immunodeficiency virus-type 1 (HIV-1) infection. Report of a Working Group of the American Academy of Neurology AIDS Task Force. Neurology 1991;41:778-785.

4 Becker JT, Martin A, Lopez OL: The dementias and AIDS; in Grant I, Martin A (eds): Neuropsychology of HIV Infection. New York, Oxford University Press, 1994, pp 133-145.

5 Antinori A, Arendt G, Becker JT, Brew BJ, Byrd DA, Cherner M, et al: Updated research nosology for HIV-associated neurocognitive disorders. Neurology 2007;69:1789-1799. 